\title{
Association between pretreatment Glasgow prognostic score and gastric cancer survival and clinicopathological features: a meta-analysis
}

This article was published in the following Dove Press journal:

OncoTargets and Therapy

27 June 2016

Number of times this article has been viewed

\author{
Chun-Xiao Zhang* \\ Shu-Yi Wang* \\ Shuang-Qian Chen \\ Shuai-Long Yang \\ Lu Wan \\ Bin Xiong
}

Department of Oncology, Zhongnan Hospital of Wuhan University, Hubei Key Laboratory of Tumor Biological Behaviors and Hubei Cancer Clinical Study Center, Wuhan, Hubei, People's Republic of China

*These authors contributed equally to this work
Correspondence: Bin Xiong

Department of Oncology, Zhongnan Hospital of Wuhan University, Hubei Key Laboratory of Tumor Biological Behaviors and Hubei Cancer Clinical Study Center, No 169 Donghu Road, Wuchang District, Wuhan 43007I, Hubei Province, People's Republic of China

Tel +86027 678I 3I52

$\mathrm{Fax}+86027678 \mid 2829$

Email binxiong1961@whu.edu.cn
Background: Glasgow prognostic score (GPS) is widely known as a systemic inflammatorybased marker. The relationship between pretreatment GPS and gastric cancer (GC) survival and clinicopathological features remains controversial. The aim of the study was to conduct a meta-analysis of published studies to evaluate the association between pretreatment GPS and survival and clinicopathological features in GC patients.

Methods: We searched PubMed, Embase, MEDLINE, and BioMed databases for relevant studies. Combined analyses were used to assess the association between pretreatment GPS and overall survival, disease-free survival, and clinicopathological parameters by Stata Version 12.0

Results: A total of 14 studies were included in this meta-analysis, including 5,579 GC patients. The results indicated that pretreatment high GPS (HGPS) predicted poor overall survival (hazard ratio $=1.51,95 \% \mathrm{CI}: 1.37-1.66, P<0.01$ ) and disease-free survival (hazard ratio $=1.45,95 \%$ CI: $1.26-1.68, P<0.01)$ in GC patients. Pretreatment HGPS was also significantly associated with advanced tumor-node-metastasis stage (odds ratio $[\mathrm{OR}]=3.09,95 \% \mathrm{CI}: 2.11-4.53, P<0.01$ ), lymph node metastasis ( $\mathrm{OR}=4.60,95 \% \mathrm{CI}: 3.23-6.56, P<0.01)$, lymphatic invasion $(\mathrm{OR}=3.04$, 95\% CI: $2.00-4.62, P<0.01$ ), and venous invasion ( $\mathrm{OR}=3.56,95 \% \mathrm{CI}: 1.81-6.99, P<0.01)$.

Conclusion: Our meta-analysis indicated that pretreatment HGPS could be a predicative factor of poor survival outcome and clinicopathological features for GC patients.

Keywords: Glasgow prognostic score, gastric cancer, survival, clinicopathological feature

\section{Introduction}

Gastric cancer (GC) is one of the most common cancers in the world. ${ }^{1}$ In recent years, although surgery, chemoradiotherapy, and targeted therapy make great progress to prolong survival of GC patients, GC is still the third leading cause of cancer-related death worldwide mainly because of tumor local recurrence and distant metastasis. ${ }^{1,2}$ Therefore, it is essential to identify GC patients with a high-risk of tumor recurrence and poor prognosis. For this purpose, both clinicians and researchers have made great efforts to seek practical biomarkers that can predict progression and survival in $\mathrm{GC}$ patients.

In the current clinical work, pathological tumor-node-metastasis (TNM) stage is a common predictive factor of predicting the prognosis and making treatment plans for GC patients, but heterogeneity of prognosis still exists in the same stage. ${ }^{3}$ This leaves a large space to search supplementary biomarkers for better predicting the prognosis of GC patients. It is increasingly recognized that variations within clinical outcomes in cancer patients were influenced by not only clinicopathological characteristics but also 
the host systemic inflammatory response. ${ }^{4}$ The connection between systemic inflammatory response and tumors has been researched in both animal models and clinical trials in the past years. ${ }^{5,6}$

Glasgow prognostic score (GPS) as an inflammationbased prognostic score is composed of serum C-reactive protein (CRP) and albumin level. The first study on GPS reported by Forrest et $\mathrm{al}^{7}$ in 2003 showed that it could be an indicator of prognosis for non-small-cell lung cancer patients. Subsequently, increasing evidence revealed that GPS acquired its prognostic significance in patients with esophageal cancer, ${ }^{8}$ pancreatic cancer, ${ }^{9}$ colorectal cancer, ${ }^{10}$ and so on. Meanwhile, increased level of GPS could predict poor survival in GC patients. ${ }^{11}$ However, due to variance in the study design, some authors did not agree with the former results. ${ }^{12}$ The impact of GPS on GC patients' prognosis still remains controversial. Thus, we searched the available studies and conducted this meta-analysis in order to evaluate the prognostic role of GPS in GC. The relationship between GPS and clinicopathological parameters was also investigated.

\section{Materials and methods Literature search}

We searched PubMed, Embase, MEDLINE, and BioMed databases for studies that assessed the prognostic role of GPS for GC patients. The keywords used for research were "Glasgow prognostic score" or "GPS", "survival" or "outcome", "clinicopathological feature", and "gastric cancer" or "stomach carcinoma". The last search was updated in December 31, 2015. Additionally, potentially related articles were also retrieved by the references in the identified articles.

\section{Study inclusion and exclusion criteria}

Inclusion criteria for selecting the articles in our analysis were as follows: 1) studies in which the patients were diagnosed as GC based on pathological examination; 2) studies that investigated the association between pretreatment GPS and clinicopathological characteristics, overall survival (OS), and disease-free survival (DFS); 3) studies that were not directly reporting hazard ratio (HR) with its $95 \%$ confidence interval (CI) were allowed if we could reconstruct HR with its $95 \%$ CI by data reported; ${ }^{13}$ and 4 ) papers that were published in English. Exclusion criteria were as follows: 1) reviews, case reports, or laboratory studies; 2) studies without available full text or sufficient data for reconstructing $\mathrm{HR}$ with its $95 \% \mathrm{CI}$; and 3 ) studies containing duplicate data.

\section{Data extraction}

For each study, the following items were extracted: 1) first author, year of publication, country of the studied population, sample size, age, predominant treatment, and follow-up period; 2) clinicopathological parameters including TNM stage, lymph node metastasis, lymphatic invasion, venous invasion, differentiated degree, and carcinoembryonic antigen level; 3) survival data including OS and DFS; and 4) the cut-off value of GPS.

\section{Quality assessment}

Two reviewers (Chun-Xiao Zhang and Shu-Yi Wang) independently implemented quality assessment in the included studies by using the Newcastle-Ottawa Quality Assessment Scale (NOS). ${ }^{14}$ The NOS comprised three parameters of quality: selection ( $0-4$ points), comparability ( $0-2$ points), and outcome assessment ( $0-3$ points). The maximum score is 9 points, and NOS scores of $\geq 6$ were assigned as high-quality studies. Any disagreement was resolved by discussion.

\section{Statistical analysis}

HR with its 95\% CI was obtained directly from each literature or estimated by data reported according to the methods illustrated by Parmar et al. ${ }^{13}$ For analyzing the correlation between GPS and clinicopathological parameters, odds ratio (OR) with its $95 \%$ CI was combined as the effective value. If both univariate and multivariate analyses were reported in the same study, multivariate analysis was chosen for the metaanalysis. Heterogeneity among pooled results was assessed using Cochran's $Q$ test and Higgins $I^{2}$ statistic. $P<0.10$ and $I^{2}>50 \%$ were considered the values that indicated significant heterogeneity, and the random-effects model was applied. If not, the fixed-effects model was performed. The potential publication bias was evaluated by Begg's funnel plot and Egger's linear regression test. The influence of publication bias on the overall effect was assessed by the "trim and fill" method described by Duval and Tweedie. ${ }^{15}$ All statistical analyses were two-sided, and the significant level was defined as $5 \%$. Statistical analyses were performed by Stata Version 12.0 (StataCorp LP, College Station, TX, USA).

\section{Results}

\section{Description of the included studies}

According to the aforementioned search terms, 132 studies were retrieved. By intensively reading the potential articles, 118 studies were excluded. Lastly, 14 studies published between 2011 and 2015 were included in this meta-analysis 
(Figure 1). ${ }^{11,12,16-27}$ Characteristics of included studies are summarized in Table 1. Fourteen retrospective studies were included containing 5,579 GC patients. Seven studies were from Japan, four from the People's Republic of China, two from Korea, and one from Italy. The sample size of included articles ranged from 102 to $1,710 \mathrm{GC}$ patients. The cut-off values of GPS were diverse, and GPS of 0 or 1 point was assigned for each CRP level less than or greater than the cut-off value ( $3 \mathrm{mg} / \mathrm{L}, 5 \mathrm{mg} / \mathrm{L}$, or $10 \mathrm{mg} / \mathrm{L}$ ) and albumin level greater than or less than the cut-off value $(35 \mathrm{~g} / \mathrm{L}$ or $38 \mathrm{~g} / \mathrm{L})$, aggregating score ranging from 0 to 2 . GPS of 2 was defined as high GPS (HGPS), and GPS of 0 or 1 was defined as low GPS (LGPS). HR with its 95\% CI was directly extracted from the original literature in 13 studies and estimated by the data reported in the remaining one study.

\section{GPS and survival outcome in GC}

There were 13 studies reporting the relationship between pretreatment GPS and OS in GC patients. ${ }^{11,12,16-22,24-27}$ With no obvious heterogeneity $\left(I^{2}=31.9 \%, P=0.13\right)$, the pooled HR of 1.51 (95\% CI: $1.37-1.66, P<0.01)$ implied that GC patients with HGPS were expected to have poor OS (Figure 2). Then, we conducted subgroup analysis according to confounders such as predominant treatment, country, sample size, and NOS score.

Stratification by predominant treatment, we found that the pooled HRs were 1.41 (95\% CI: $1.26-1.58, P<0.01)$

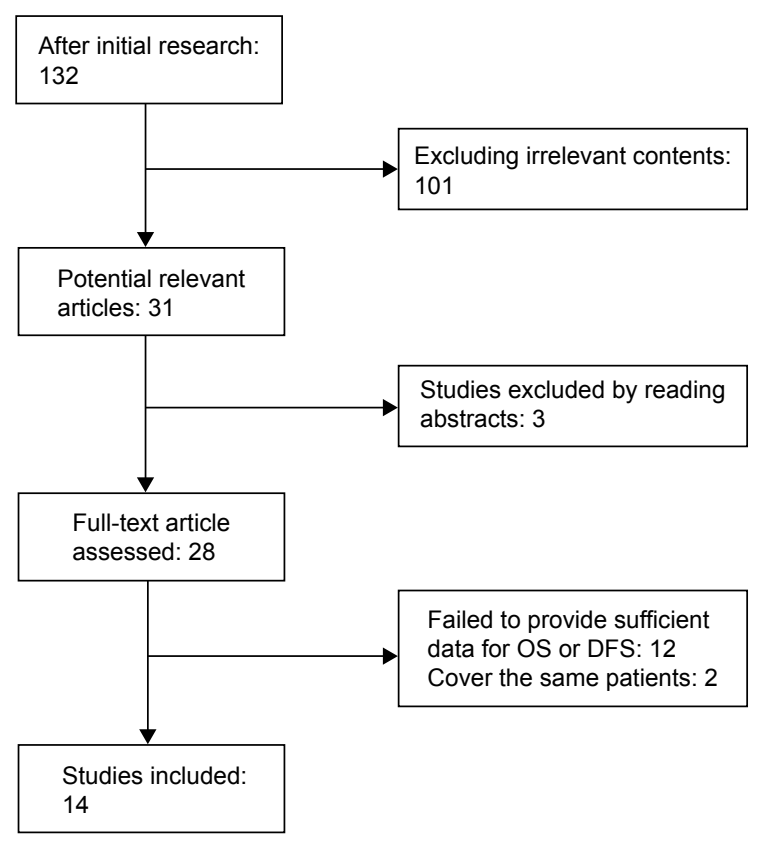

Figure I The flow chart of study selection. Abbreviations: DFS, disease-free survival; OS, overall survival. for patients treated by surgery and 1.78 (95\% CI: 1.48-2.13, $P<0.01)$ for patients treated by chemotherapy. Subgroup analyses by country indicated that HGPS predicted poor prognosis for patients both in Japan (HR $=1.57$, 95\% CI: $1.28-1.93, P<0.01)$ and in other countries $(\mathrm{HR}=1.49$, 95\% CI: $1.33-1.66, P<0.01)$. In addition, subgroup analysis showed that HGPS could also predict poor prognosis for GC regardless of sample size $(<300$ vs $\geq 300)$ and NOS score $(<7$ vs $\geq 7$ ) (Table 2$)$.

There were four studies reporting the relationship between pretreatment GPS and DFS in GC patients. ${ }^{16,23,25,27}$ The pooled result ( $\mathrm{HR}=1.45,95 \% \mathrm{CI}: 1.26-1.68, P<0.01$ ) showed significant correlation between HGPS and short DFS with no heterogeneity $\left(I^{2}=0 \%, P=0.46\right.$; Figure 3$)$.

\section{GPS and clinicopathological features in GC}

Six studies presented the relationship between pretreatment GPS and TNM stage in GC patients. ${ }^{11,12,17,19,23,24}$ The combined OR of 3.09 (95\% CI: 2.11-4.53, $P<0.01)$ displayed that HGPS was associated with advanced TNM stage for GC patients, with no significant heterogeneity $\left(I^{2}=46.9 \%\right.$, $P=0.09$; Figure 4A).

Five studies presented the relationship between pretreatment GPS and lymph node metastasis in GC patients. ${ }^{12,17,19,23,24}$ The combined OR of 4.60 (95\% CI: 3.23-6.56, $P<0.01$ ) displayed that GC patients with HGPS were prone to lymph node metastasis with no obvious heterogeneity $\left(I^{2}=45.4 \%\right.$, $P=0.12$; Figure 4B).

There were five studies reporting the relationship between pretreatment GPS and lymphatic invasion in GC patients. ${ }^{12,17,19,23,24}$ The pooled estimate (OR $=3.04,95 \%$ CI: $2.00-4.62, P<0.01)$ showed that HGPS was markedly associated with lymphatic invasion with minor heterogeneity $\left(I^{2}=36.5 \%, P=0.18\right.$; Figure 4C).

Five studies showed the connection between pretreatment GPS and venous invasion in GC patients. ${ }^{12,17,19,23,24}$ Although with heterogeneity $\left(I^{2}=71.3 \%, P<0.01\right)$, the combined result $(\mathrm{OR}=3.56,95 \% \mathrm{CI}: 1.81-6.99, P<0.01)$ revealed an obvious relevance between HGPS and venous invasion (Figure 4D).

No statistically significant difference was found in the relationship between pretreatment GPS and differentiated degree (poor vs well or moderate) and carcinoembryonic antigen level ( $\geq 5 \mathrm{ng} \mathrm{mL}^{-1}$ vs $<5 \mathrm{ng} \mathrm{mL}^{-1}$ ) (Table 3 ).

\section{Publication bias}

We evaluated publication bias using Begg's funnel plot and Egger's linear regression test. Publication bias was found 


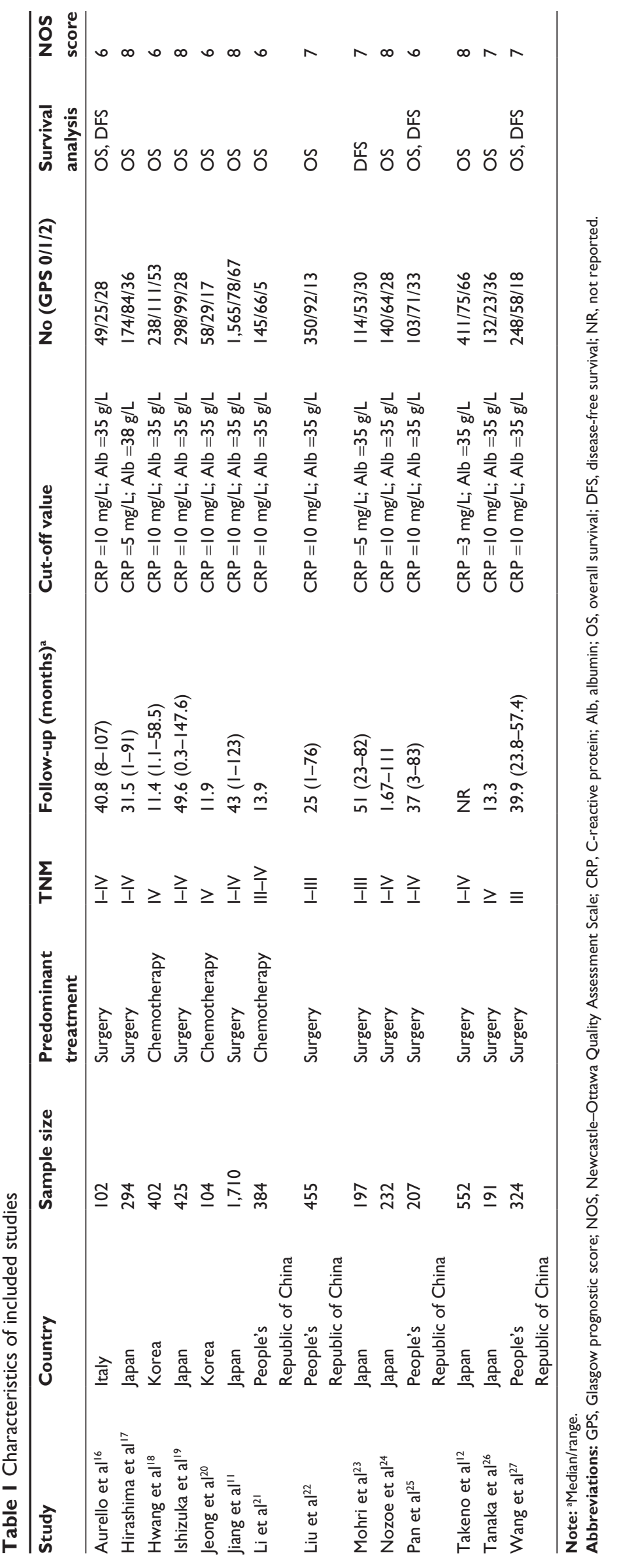




\begin{tabular}{|c|c|c|}
\hline Study ID & HR $(95 \% \mathrm{Cl})$ & Weight (\%) \\
\hline Aurello et al ${ }^{16}$ & $1.91(1.38,3.18)$ & 5.30 \\
\hline Hirashima et al ${ }^{17}$ & $2.26(1.09,4.69)$ & 1.74 \\
\hline Hwang et al ${ }^{18}$ & $1.76(1.41,2.19)$ & 19.06 \\
\hline Ishizuka et al ${ }^{19}$ & $2.05(1.00,4.18)$ & 1.81 \\
\hline Jeong et $\mathrm{al}^{20}$ & $2.29(1.21,4.32)$ & 2.28 \\
\hline Jiang et al ${ }^{11}$ & $1.85(1.18,2.88)$ & 4.70 \\
\hline Li et $\mathrm{al}^{21}$ & $1.68(1.12,2.34)$ & 6.88 \\
\hline Liu et $\mathrm{al}^{22}$ & $1.16(0.91,1.48)$ & 15.63 \\
\hline Nozoe et $\mathrm{al}^{24}$ & $3.42(1.21,9.71)$ & 0.85 \\
\hline Pan et $\mathrm{al}^{25}$ & $1.33(1.04,1.70)$ & 15.56 \\
\hline Takeno et al ${ }^{12}$ & $1.24(0.92,1.68)$ & 10.17 \\
\hline Tanaka et al ${ }^{26}$ & $1.53(0.88,2.66)$ & 3.02 \\
\hline Wang et $\mathrm{al}^{27}$ & $1.40(1.07,1.82)$ & 12.99 \\
\hline Overall $\left(I^{2}=31.9 \%, P=0.128\right)$ & $1.51(1.37,1.66)$ & 100 \\
\hline
\end{tabular}

Figure 2 The forest plot between pretreatment GPS and OS in GC patients.

Abbreviations: GPS, Glasgow prognostic score; OS, overall survival; GC, gastric cancer; HR, hazard ratio; Cl, confidence interval; HGPS, high Glasgow prognostic score; LGPS, low Glasgow prognostic score.

Table 2 Results of subgroup analysis

\begin{tabular}{|c|c|c|c|c|c|c|c|}
\hline \multirow[t]{2}{*}{ Subgroup } & \multirow{2}{*}{$\begin{array}{l}\text { No of } \\
\text { studies }\end{array}$} & \multicolumn{2}{|c|}{ Random-effects model } & \multicolumn{2}{|c|}{ Fixed-effects model } & \multicolumn{2}{|c|}{ Heterogeneity } \\
\hline & & HR (95\% Cl) & $P$-value & HR (95\% Cl) & $P$-value & $I^{2}(\%)$ & $P$-value \\
\hline \multicolumn{8}{|c|}{ Predominant treatment } \\
\hline Surgery & 10 & $1.46(1.26-1.68)$ & $<0.01$ & $1.41(1.26-1.58)$ & $<0.01$ & 27.3 & 0.19 \\
\hline Chemotherapy & 3 & $1.78(1.48-2.13)$ & $<0.01$ & $1.78(1.48-2.13)$ & $<0.01$ & 0.0 & 0.70 \\
\hline \multicolumn{8}{|l|}{ Country } \\
\hline Japan & 6 & $1.66(1.29-2.14)$ & $<0.01$ & $1.57(1.28-1.93)$ & $<0.01$ & 23.4 & 0.26 \\
\hline Other & 7 & $1.51(1.30-1.77)$ & $<0.01$ & $1.49(1.33-1.66)$ & $<0.01$ & 44.5 & 0.09 \\
\hline \multicolumn{8}{|l|}{ Sample size } \\
\hline$<300$ & 6 & $1.73(1.35-2.20)$ & $<0.01$ & $1.60(1.34-1.92)$ & $<0.01$ & 28.7 & 0.22 \\
\hline$\geq 300$ & 7 & $1.48(1.27-1.73)$ & $<0.01$ & $1.47(1.31-1.65)$ & $<0.01$ & 39.5 & 0.13 \\
\hline \multicolumn{8}{|l|}{ NOS score } \\
\hline$<7$ & 5 & $1.65(1.41-1.92)$ & $<0.01$ & $1.64(1.42-1.88)$ & $<0.01$ & 16.2 & 0.31 \\
\hline$\geq 7$ & 8 & $1.46(1.22-1.74)$ & $<0.01$ & $1.39(1.22-1.59)$ & $<0.01$ & 30.6 & 0.18 \\
\hline
\end{tabular}

Abbreviations: $\mathrm{HR}$, hazard ratio; $\mathrm{Cl}$, confidence interval; NOS, Newcastle-Ottawa Quality Assessment Scale.

\begin{tabular}{|c|c|c|}
\hline Study ID & HR (95\% CI) & Weight (\%) \\
\hline Aurello et $\mathrm{al}^{16}$ & $1.73(1.16,2.39)$ & 16.38 \\
\hline Mohri et $\mathrm{al}^{23}$ & $1.81(1.12,2.96)$ & 9.06 \\
\hline Pan et $\mathrm{al}^{25}$ & $1.29(1.01,1.65)$ & 36.26 \\
\hline Wang et $\mathrm{al}^{27}$ & $1.43(1.13,1.81)$ & 38.29 \\
\hline Overall $\left(I^{2}=0.0 \%, P=0.462\right)$ & $1.45(1.26,1.68)$ & 100 \\
\hline
\end{tabular}

Figure 3 The forest plot between pretreatment GPS and DFS in GC patients.

Abbreviations: GPS, Glasgow prognostic score; DFS, disease-free survival; GC, gastric cancer; HR, hazard ratio; Cl, confidence interval; HGPS, high Glasgow prognostic score; LGPS, low Glasgow prognostic score. 


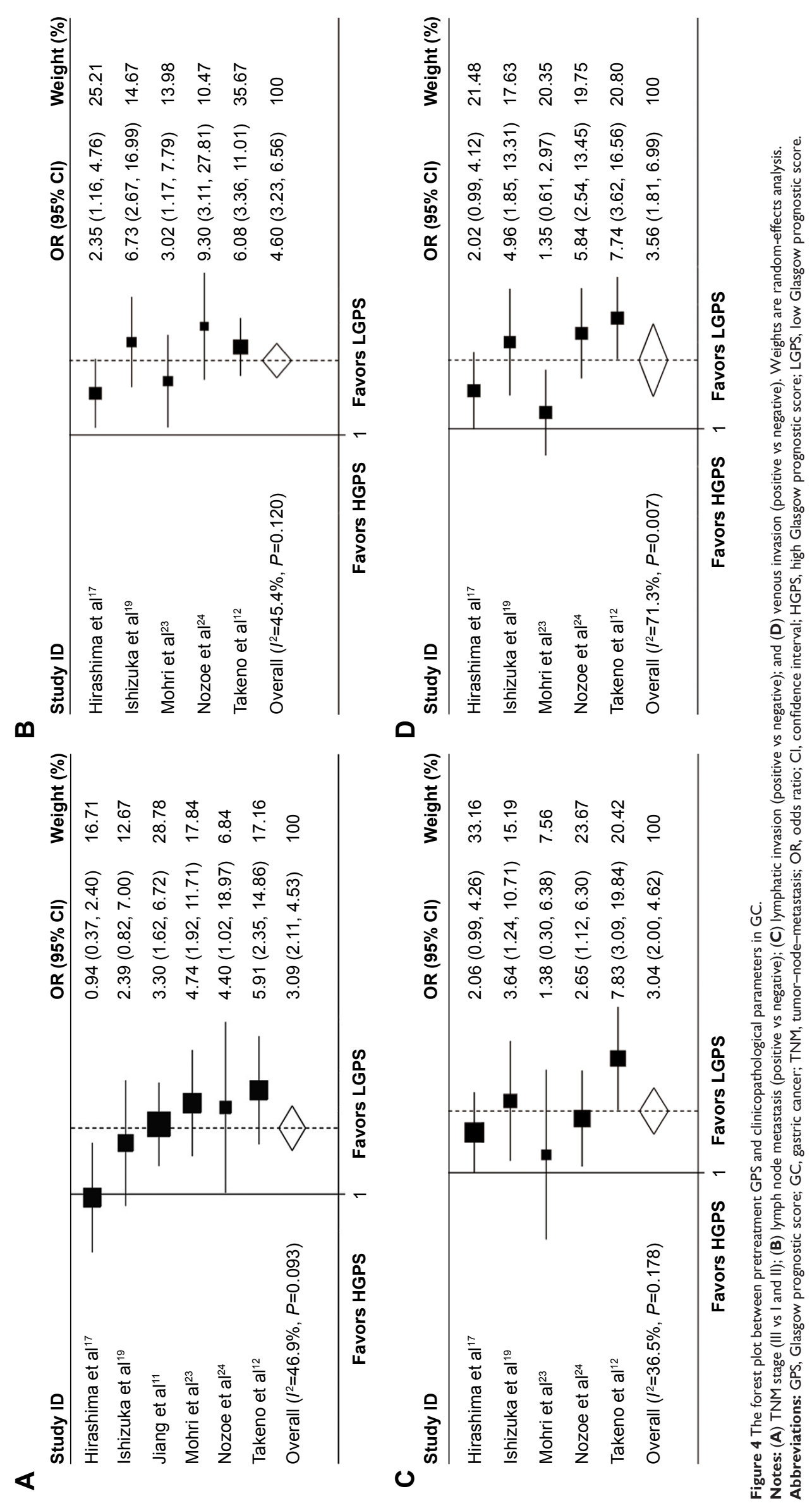


Table 3 Relationship between pretreatment GPS and differentiated degree and CEA level

\begin{tabular}{llll}
\hline Factors & OR & $\mathbf{9 5 \%} \mathbf{C l}$ & P-value \\
\hline Differentiated degree $^{\mathrm{a}}$ & 1.02 & $0.89-1.17$ & 0.79 \\
CEA level $^{\mathrm{b}}$ & 2.13 & $0.5 \mathrm{I}-8.87$ & 0.30 \\
\hline
\end{tabular}

Notes: aPoor vs well or moderate, ${ }^{b} \geq 5 \mathrm{ng} \mathrm{mL}^{-1}$ vs $<5 \mathrm{ng} \mathrm{mL}^{-1}$.

Abbreviations: GPS, Glasgow prognostic score; CEA, carcinoembryonic antigen; $\mathrm{OR}$, odds ratio; $\mathrm{Cl}$, confidence interval.

only in OS ( $P=0.04$ for Begg's test and $P=0.02$ for Egger's test; Figure 5A). Therefore, the "trim and fill" analysis was further performed. As a result, there were four studies hypothetically remained unpublished, and recalculated result did not change significantly ( $\mathrm{HR}=1.46,95 \% \mathrm{CI}: 1.33-1.60$, $P<0.01$ ), indicating the stability of the result (Figure 5B).

\section{Discussion}

Systemic inflammatory response played a crucial role in the development and progression of various tumors. ${ }^{28}$ Several biomarkers based on inflammation including GPS, ${ }^{29}$ neutrophil-lymphocyte ratio,,$^{30}$ and platelet-lymphocyte ratio $^{31}$ had been reported to be independent prognostic predictors for cancer patients. However, it was not clear which of these biomarkers better predicted survival for GC patients.

Many recent studies indicated that pretreatment GPS was superior to other biomarkers in predicting survival in either operable GC patients ${ }^{16,27}$ or inoperable advanced GC patients. ${ }^{20,21}$ Besides, GPS, which is easily obtained by peripheral blood test before treatment, can give a preoperative risk assessment, whereas TNM stage and lymphovascular invasion give a postoperative risk assessment. Preoperative HGPS would identify patients at high risk of tumor recurrence and poor prognosis, helping clinicians to focus on these patients who would need more careful TNM stage, rigorous

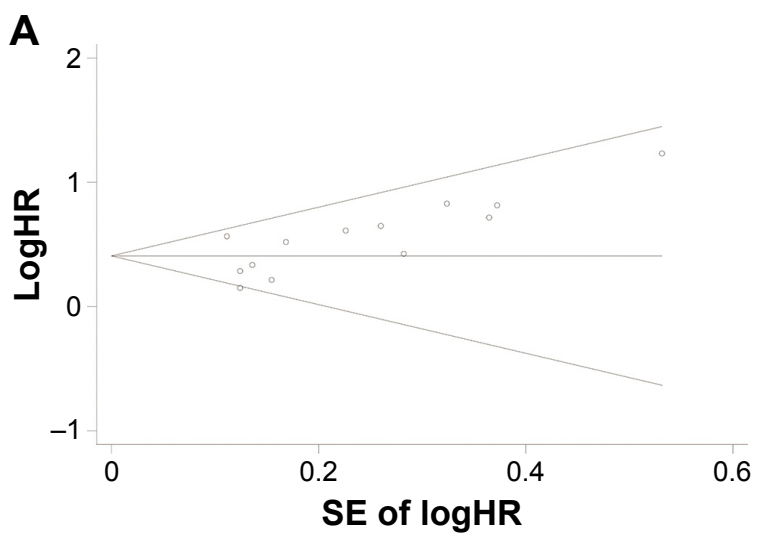

follow-up, and imperative adjuvant treatment. Pan et $\mathrm{a}^{25}$ showed that adjuvant chemotherapy after resection could significantly prolong OS and DFS in GC patients with LGPS, but not with HGPS. The result was similar to the study of Wang et $\mathrm{al}^{27}$ who reported that in stage III GC patients receiving adjuvant chemotherapy, there was a significant decrease in the median survival for those patients with HGPS comparing with those patients with LGPS, which suggested that patients with HGPS derived little survival benefit from active treatment. Therefore, GPS could not only simply represent the host systemic inflammatory response but also importantly be used to predict survival outcome and to individualize treatment plans for GC patients.

The results of our study revealed that pretreatment HGPS could predict poor OS and DFS in GC patients. Meanwhile, there was an obvious relationship between pretreatment HGPS and clinicopathological parameters, such as TNM stage, lymph node metastasis, lymphatic invasion, and venous invasion. According to the above-mentioned results, GPS is a promising prognostic biomarker of GC patients assisting in predicting prognosis and identifying treatment plans.

There were some limitations to this meta-analysis. First, language selection may bring bias. We have restricted our analysis to published studies written in English, other languages such as Japanese and Korean were excluded based on language criteria. This may result in language bias leading to an overestimation of effect sizes. ${ }^{32}$ Second, heterogeneity among included studies was observed in the results. This may be because of confounding factors, such as different characteristics of enrolled patients, sample size, treatment method, follow-up period, and cut-off value of GPS. We handled the issue of heterogeneity by a rigorous methodological approach that utilized random-effects model for more

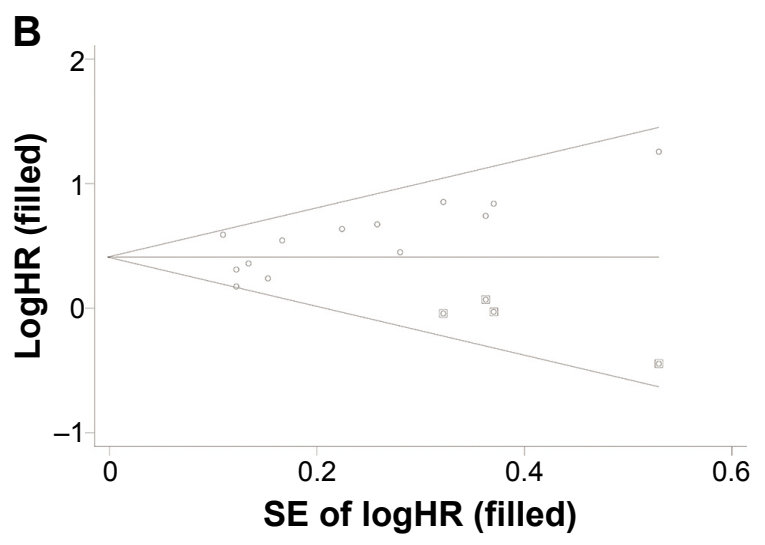

Figure 5 Funnel plot of included studies reporting OS in GC patients.

Notes: (A) Begg's funnel plot for the assessment of potential publication bias; (B) funnel plot adjusted with trim and fill method. Circles: included studies. Squares: presumed missing studies.

Abbreviations: OS, overall survival; GC, gastric cancer; HR, hazard ratio; SE, standard error. 
conservative estimate. Moreover, the funnel plot analysis of OS showed some asymmetry, and publication bias was confirmed by Begg's test and Egger's test. Thus, the pooled result may be somehow overvalued. But the followed "trim and fill" analysis did not change the general result, which further reinforced the prognostic role of GPS in GC patients.

\section{Conclusion}

Our meta-analysis indicated that pretreatment HGPS, namely elevated CRP and reduced albumin level, was associated with poor survival and clinicopathological features in GC. It might be used as a prognostic biomarker for GC and figure out the high-risk patients who may not benefit from the antitumor therapies and consequently adjust treatment strategies. However, large-scale prospective investigations need to be conducted to better understand the prognostic role of GPS in GC patients.

\section{Disclosure}

The authors report no conflicts of interest in this work.

\section{References}

1. Torre LA, Bray F, Siegel RL, Ferlay J, Lortet-Tieulent J, Jemal A. Global cancer statistics, 2012. Ca-Cancer J Clin. 2015;65(2):87-108.

2. Maehara Y, Kakeji Y, Oda S, Takahashi I, Akazawa K, Sugimachi K. Time trends of surgical treatment and the prognosis for Japanese patients with gastric cancer. Brit J Cancer. 2000;83(8):986-991.

3. Peng CW, Wang LW, Zeng WJ, Yang XJ, Li Y. Evaluation of the staging systems for gastric cancer. J Surg Oncol. 2013;108(2):93-105.

4. MacDonald N. Cancer cachexia and targeting chronic inflammation: a unified approach to cancer treatment and palliative/supportive care. J Support Oncol. 2007;5(4):157-162; discussion 164-156, 183.

5. Coussens LM, Werb Z. Inflammation and cancer. Nature. 2002; 420(6917):860-867.

6. Grivennikov SI, Greten FR, Karin M. Immunity, inflammation, and cancer. Cell. 2010;140(6):883-899.

7. Forrest LM, McMillan DC, McArdle CS, Angerson WJ, Dunlop DJ. Evaluation of cumulative prognostic scores based on the systemic inflammatory response in patients with inoperable non-small-cell lung cancer. Brit J Cancer. 2003;89(6):1028-1030.

8. Crumley ABC, McMillan DC, McKernan M, McDonald AC, Stuart RC. Evaluation of an inflammation-based prognostic score in patients with inoperable gastro-oesophageal cancer. Brit J Cancer. 2006;94(5): 637-641.

9. Glen P, Jamieson NB, McMillan DC, Carter R, Imrie CW, McKay CJ. Evaluation of an inflammation-based prognostic score in patients with inoperable pancreatic cancer. Pancreatology. 2006;6(5):450-453.

10. McMillan DC, Crozier JEM, Canna K, Angerson WJ, McArdle CS. Evaluation of an inflammation-based prognostic score (GPS) in patients undergoing resection for colon and rectal cancer. Int J Colorectal Dis. 2007;22(8):881-886.

11. Jiang X, Hiki N, Nunobe S, et al. Prognostic importance of the inflammation-based Glasgow prognostic score in patients with gastric cancer. Brit J Cancer. 2012;107(2):275-279.

12. Takeno S, Hashimoto T, Shibata R, et al. The high-sensitivity modified Glasgow prognostic score is superior to the modified Glasgow prognostic score as a prognostic predictor in patients with resectable gastric cancer. Oncology-Basel. 2014;87(4):205-214.
13. Parmar MKB, Torri V, Stewart L. Extracting summary statistics to perform meta-analyses of the published literature for survival endpoints. Stat Med. 1998;17(24):2815-2834.

14. Stang A. Critical evaluation of the Newcastle-Ottawa scale for the assessment of the quality of nonrandomized studies in meta-analyses. Eur J Epidemiol. 2010;25(9):603-605.

15. Duval S, Tweedie R. Trim and fill: a simple funnel-plot-based method of testing and adjusting for publication bias in meta-analysis. Biometrics. 2000;56(2):455-463.

16. Aurello P, Tierno SM, Berardi G, et al. Value of preoperative inflammation-based prognostic scores in predicting overall survival and disease-free survival in patients with gastric cancer. Ann Surg Oncol. 2014;21(6):1998-2004.

17. Hirashima K, Watanabe M, Shigaki H, et al. Prognostic significance of the modified Glasgow prognostic score in elderly patients with gastric cancer. J Gastroenterol. 2014;49(6):1040-1046.

18. Hwang JE, Kim HN, Kim DE, et al. Prognostic significance of a systemic inflammatory response in patients receiving first-line palliative chemotherapy for recurred or metastatic gastric cancer. BMC Cancer. 2011; $11: 489$.

19. Ishizuka M, Oyama Y, Abe A, Tago K, Tanaka G, Kubota K. Clinical significance of an inflammation-based prognostic system for gastric cancer patients with a preoperative normal serum level of carcinoembryonic antigen. Anticancer Res. 2014;34(12):7219-7226.

20. Jeong JH, Lim SM, Yun JY, et al. Comparison of two inflammationbased prognostic scores in patients with unresectable advanced gastric cancer. Oncology-Basel. 2012;83(5):292-299.

21. Li QQ, Lu ZH, Yang L, et al. Neutrophil count and the inflammationbased Glasgow prognostic score predict survival in patients with advanced gastric cancer receiving first-line chemotherapy. Asian PacJ Cancer Prev. 2014;15(2):945-950.

22. Liu XC, Sun XW, Liu JJ, et al. Preoperative C-reactive protein/albumin ratio predicts prognosis of patients after curative resection for gastric cancer. Transl Oncol. 2015;8(4):339-345.

23. Mohri Y, Tanaka K, Ohi M, et al. Inflammation-based prognostic score as a predictor of postoperative gastric cancer recurrence. Anticancer Res. 2012;32(10):4581-4584.

24. Nozoe T, Iguchi T, Egashira A, Adachi E, Matsukuma A, Ezaki T. Significance of modified Glasgow prognostic score as a useful indicator for prognosis of patients with gastric carcinoma. Am J Surg. 2011;201(2): 186-191.

25. Pan QX, Su ZJ, Zhang JH, Wang CR, Ke SY. A comparison of the prognostic value of preoperative inflammation-based scores and TNM stage in patients with gastric cancer. Onco Targets Ther. 2015;8: 1375-1385.

26. Tanaka H, Muguruma K, Toyokawa T, Kubo N, Ohira M, Hirakawa K. Differential impact of the neutrophil-lymphocyte ratio on the survival of patients with stage IV gastric cancer. Digest Surg. 2014;31(4-5): 327-333.

27. Wang DS, Ren C, Qiu MZ, et al. Comparison of the prognostic value of various preoperative inflammation-based factors in patients with stage III gastric cancer. Tumor Biol. 2012;33(3):749-756.

28. Hanahan D, Weinberg RA. Hallmarks of cancer: the next generation. Cell. 2011;144(5):646-674.

29. McMillan DC. The systemic inflammation-based Glasgow prognostic score: a decade of experience in patients with cancer. Cancer Treat Rev. 2013;39(5):534-540.

30. Guthrie GJK, Roxburgh CSD, Horgan PG, McMillan DC. Does interleukin-6 link explain the link between tumour necrosis, local and systemic inflammatory responses and outcome in patients with colorectal cancer? Cancer Treat Rev. 2013;39(1):89-96.

31. Zhou X, Du YP, Huang ZB, et al. Prognostic value of PLR in various cancers: a meta-analysis. PLoS One. 2014;9(6):e101119.

32. Pham B, Klassen TP, Lawson ML, Moher D. Language of publication restrictions in systematic reviews gave different results depending on whether the intervention was conventional or complementary. J Clin Epidemiol. 2005;58(8):769-776. 
OncoTargets and Therapy

\section{Publish your work in this journal}

OncoTargets and Therapy is an international, peer-reviewed, open access journal focusing on the pathological basis of all cancers, potential targets for therapy and treatment protocols employed to improve the management of cancer patients. The journal also focuses on the impact of management programs and new therapeutic agents and protocols on

patient perspectives such as quality of life, adherence and satisfaction. The manuscript management system is completely online and includes a very quick and fair peer-review system, which is all easy to use. Visit http://www.dovepress.com/testimonials.php to read real quotes from published authors.

Submit your manuscript here: http://www.dovepress.com/oncotargets-and-therapy-journal 